\title{
Pyrexia of unknown origin in association with bladder diverticulum tumour
}

\author{
M. Agarwal, A. Azzopardi and G.R. Mufti \\ Department of Urology, St Bartholomew's Hospital, Rochester, Kent ME1 IDS, UK
}

Summary: A case of pyrexia due to a rapidly growing undifferentiated transitional cell carcinoma in a bladder diverticulum is described. The literature is reviewed and the causes of pyrexia associated with neoplasia briefly discussed.

\section{Introduction}

Tumours are known to be associated with pyrexia, some more commonly than others. Recently we were involved with the management of a patient with pyrexia due to undifferentiated bladder carcinoma. Literature survey revealed only two such cases published before. The extreme rarity of this occurrence prompted us to report this case.

\section{Case report}

A 56 year old Asian male was admitted with a sole symptom of painless haematuria of 2 weeks duration. Intravenous urography revealed normal upper tracts and a large bladder diverticulum. Bacteriological examination of urine did not reveal any evidence of infection. Urine cytology was positive for malignant cells. Intravenous urography demonstrated normal upper tracts and a large bladder diverticulum. Cystoscopy revealed a solid tumour within the diverticulum which was resected completely down to the base. The rest of the bladder was normal. Histological examination showed this to be G3 transitional cell carcinoma with glandular differentiation, infiltrating the lamina propria.

Six weeks later he was readmitted for cystoscopy. The tumour had recurred and it was resected again. The histological appearances were similar to the first resection. During this admission too there was no evidence of infection on bacteriological examination of urine. The patient was discharged and referred for radical radiotherapy.

Correspondence: G.R. Mufti, M.S., M.Ch., F.R.C.S. (Ed).

Accepted: 2 November 1992
Two weeks later (before radiotherapy could be commenced) he was readmitted with a spiking temperature $\left(38-40.5^{\circ} \mathrm{C}\right)$ with a polymorphonuclear leucocytosis $\left(15-23 \times 10^{9} / 1\right)$. Repeated urine and blood cultures were negative for infection. Extensive investigations to locate an infective source were negative. In view of the ethnic background of the patient rare causes of pyrexia of unknown origin like malaria and typhoid fever were also excluded. Ultrasound scan and subsequent computed tomographic scan of the pelvis demonstrated a nonhomogenous solid mass in the bladder diverticulum (Figure 1); there was no evidence of pelvic or abdominal metastases. Isotope bone scan was normal. After 3 weeks of persistent fever and fruitless investigation laparotomy was undertaken. There was no evidence of pelvic or abdominal lymphadenopathy, the liver was normal. Excision of the bladder diverticulum with a wide margin of normal bladder wall was performed. Pyrexia returned to normal within 24 hours of the operation.

Histological examination of the excised specimen revealed a solid tumour within the diverticulum (Figure 2). There was no evidence of sepsis within the diverticular sac. Microscopy showed infiltrating G3 transitional cell carcinoma; extensive areas of tumour necrosis which could have accounted for the pyrexia were not present in any of the sections examined. The external surface of the diverticulum and the edges of the excised bladder wall were free of tumour. The postoperative period was uneventful and the patient was discharged 10 days after the surgical procedure. He was referred for radical radiotherapy which was due to commence 6 weeks after discharge.

A fortnight before the commencement date of 


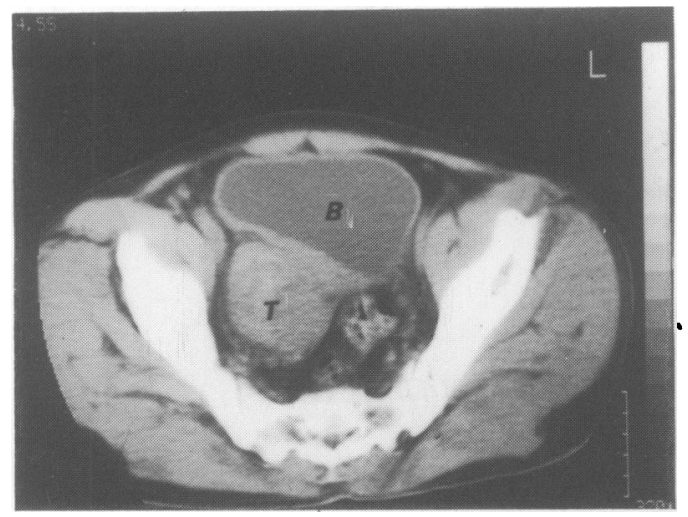

Figure 1 Computed tomographic scan showing tumour in bladder diverticulum. $\mathbf{B}=$ bladder; $\mathrm{T}=$ diverticulum tumour.

radiotherapy the patient was readmitted with a swinging pyrexia $\left(38.5-40.5^{\circ} \mathrm{C}\right)$ associated with a polymorphonuclear leucocytosis $\left(18-23 \times 10^{9} / 1\right)$. On this occasion as well repeated urine and blood cultures were free of infection. He was again investigated for 2 weeks to locate an infected focus but without success. However, a repeat computed tomographic scan of the pelvis revealed a large nonhomogeneous mass behind the bladder. Since the pyrexia did not settle a repeat laparotomy was undertaken which showed a large recurrence behind the bladder on the right side with bilateral iliac and para-aortic lymphadenopathy. Biopsies of the recurrence and lymph node confirmed the diagnosis of undifferentiated transitional cell carcinoma of bladder. There was no evidence of extensive areas of tumour necrosis in any of the sections examined. Postoperatively the pyrexia settled for 3 days before recurring again. The patient died on the 10th postoperative day.

\section{Discussion}

Any malignant neoplasm can lead to pyrexia due to secondary infection. Thus $10 \%$ of colorectal carcinomas are associated with pyrexia due to pericolonic abscesses or abscess formation within the intraluminal polyp. ${ }^{1}$ Similarly carcinoma lung can present with pyrexia owing to secondary atelectasis or pneumonia. However, pyrexia due to the neoplastic process itself is commonly associated

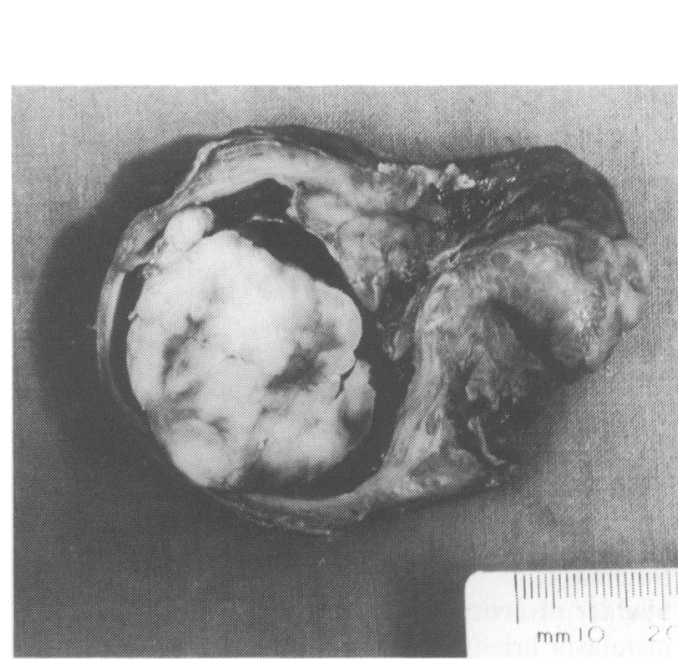

Figure 2 Excised specimen showing a solid tumour. within the bladder diverticulum.

with lymphoreticular tumours, namely Hodgkin's and non-Hodgkin's lymphomas, acute and chronic ${ }_{\circ}$ myelocytic leukaemias, and acute lymphatic $\stackrel{ }{-}$ leukaemia. ${ }^{2}$ Renal cell carcinoma is a classical $\vec{z}$ malignant cause of pyrexia of unknown origin. It has been suggested that the tumour cells secrete the endogenous pyrogen. ${ }^{3}$ Occasionally benign lesiogs? like leiomyomas of uterus, stomach or intestipe ${ }^{\omega}$ produce fever due to the degeneration and necrosids of tumour cells.

Pyrexia associated with bladder carcinoma is extremely rare. On literature survey we could find only two such published accounts. In one patient $\stackrel{\varnothing}{\varrho}$ pyrexia was secondary to upper renal tract obstruc $-\overrightarrow{\overrightarrow{0}}$ tion and infection. ${ }^{4}$ In the second case the presenta- $\frac{0}{3}$ tion was somewhat similar to that of our patient in that the fever was due to the neoplastic processo itself. ${ }^{5}$ Furthermore, in their case as in ours the temperature returned to normal after open excision 3 . of the tumour. The notable feature in our patient was the extreme rapidity of the tumour growth and 3 . the fulminating course of the disease. It is difficulto to say, in retrospect, whether or not parenteral chemotherapy started soon after the first laparo-응 tomy would have made any difference to the course $>$ of the disease or the eventual outcome.

\section{Acknowledgement}

We are grateful to Miss Tina Anderson for: secretarial assistance. 
1. Aderka, D., Hausmann, M., Santo, M. et al. Unexplained episodes of fever: an early manifestation of colorectal carcinoma. Israeli J Med Sci 1989, 121: 421-424.

2. Brusch, J.L. \& Wemstein, L. Fever of unknown origin. Med Clin North Am 1988, 72: 1247-1261.

3. Rawlins, M.P., Luff, R.H. \& Cranston, W.I. Pyrexia in renal carcinoma. Lancet 1970, 1: 1371-1373.
4. Saa-Gandi, F.W., Newling, D.W.W. \& Cook, A.M. Pyrexia of unknown origin. An atypical presentation of bladder carcinoma. Br J Urol 1989, 64: 548.

5. Moulonguet, A. \& Camplez, P. Bladder cancer with fever as the first symptom. J Urol Nephrol 1971, 77: 473-476.

\title{
Invasive aspergillosis in two patients with acute lymphoblastic leukaemia in complete remission
}

\author{
Marc L. Turner, Lesley Russell, Leslie J.R. Milne ${ }^{1}$ and Alistair C. Parker \\ Department of Haematology, Royal Infirmary of Edinburgh, Lauriston Place, Edinburgh EH3 9YW and \\ ${ }^{1}$ Mycology Unit, Western General Hospital, Edinburgh, UK
}

\begin{abstract}
Summary: Invasive aspergillosis is a disease of the immunosuppressed patient. We describe two patients with acute lymphoblastic leukaemia who attained complete remission, with partial or complete bone marrow recovery, but who went on to develop fatal invasive aspergillosis contemporaneous with recovery of neutrophil counts. Quantitative recovery of peripheral blood neutrophil counts does not guarantee control of Aspergillus infection, perhaps due to functional neutrophil deficiencies postchemotherapy, and specific defensive strategies adopted by the organism itself.
\end{abstract}

\section{Introduction}

Aspergillus spp. can cause a spectrum of disease in man, including allergic bronchopulmonary aspergillosis, and formation of aspergilloma in patients with pre-existing upper or lower respiratory tract disease. During neutropenia and other states of immunocompromise, Aspergillus spp. can become invasive, leading to local tissue destruction, haemorrhage, thrombosis, and/or widespread haematogenous dissemination. Invasive aspergillosis poses serious diagnostic and therapeutic problems, and is associated with a high morbidity and mortality.

We describe two patients with acute lymphoblastic leukaemia, who attained complete remission following inductive chemotherapy. These cases are remarkable in that invasive aspergillosis developed contemporaneously with recovery of bone marrow function, and underwent fatal progression despite normal peripheral blood neutrophil counts.

Correspondence: Marc L. Turner, M.R.C.P.

Accepted: 25 September 1992

\section{Case reports}

Case 1

A 51 year old female presented in August 1989 with acute lymphoblastic leukaemia of $\mathrm{T}$ cell lineage. There was no evidence of central nervous system involvement, and she received induction chemotherapy according to UKALL Xa protocol (Table I). She was nursed in a single room without laminar air flow. Complete remission was achieved without incident. In mid-September 1989 she received early intensification (Table I), and during the ensuing period of neutropenia developed oral and oesophageal candidosis, which responded to oral fluconazole.

At the beginning of October 1989 the patient complained of pain around the medial aspect of the left orbit, overlying the left ethmoid sinus. The peripheral blood counts had partially recovered, but over the next 2-3 days she developed left proptosis, with an ipsilateral VIth and sympathetic palsy. The condition progressed to a complete external and internal ophthalmoplegia, and a central field defect. Computed tomography (CT) scan 